\title{
REDIMENSIONAMENTO DO SISTEMA DE RESFRIAMENTO DA FERMENTAÇÃO PARA ELEVAÇÃO DA CAPACIDADE DE PRODUÇÃO DE ETANOL
}

\author{
A. I. C. GARNICA, J. E. M. PAZ, F. D. S. CURBELO \\ Universidade Federal da Paraíba \\ brasil@ifrn.edu.br*
}

Artigo submetido em 07/03/2019 e aceito em 09/08/2019

DOI: $10.15628 /$ holos.2019.8374

\section{RESUMO}

O Brasil é um dos maiores produtores de cana-de-açúcar do mundo, produzindo através dela mais de 37 milhões de toneladas de açúcar e 27 bilhões de litros de etanol. Atualmente há uma elevação na demanda do consumo de etanol combustível, especialmente em função da variação do preço do petróleo no mercado internacional e o caráter renovável desta fonte de energia. Este trabalho tem como objetivo elevar a produção de etanol a partir do redimensionamento do sistema de resfriamento da fermentação de uma planta industrial, para tal feito foi necessário realizar um balanço de energia do sistema de arrefecimento da fermentação, em sua antiga configuração, e redistribuir as placas para atender a necessidade de absorção de calor do primeiro e segundo estágios. Outro ponto importante foi a ampliação do segundo estágio, aumentando o tempo de
\end{abstract}

residência do mosto fermentado. As modificações implementadas geraram um aumento de produtividade da planta mensurados através da quantidade de cana de açúcar destinada a produção de etanol que dobrou em relação a safra anterior, a quantidade de vinho produzido que foi $36 \%$ maior, a elevação de $0,9 \%$ na quantidade de açúcares redutores totais (ART) presentes na alimentação, o que elevou em $6 \%$ o teor alcoólico do vinho, proporcionando uma produção $52 \%$ maior no período estudado, mantendo a temperatura da fermentação na faixa ótima de trabalho da levedura. Estes resultados evidenciam a elevação na produtividade do sistema e ainda proporcionou uma redução no custo de manutenção através da redução do número de trocadores de calor e bombas em operação na planta.

PALAVRAS-CHAVE: Balanço de energia, Fermentação alcoólica, Trocadores de calor de placas.

\section{REDIMENSIONING THE FERMENTATION COOLING SYSTEM FOR EFFECT OF ETHANOL PRODUCTION CAPACITY}

\section{ABSTRACT}

Brazil is one of the largest producers of sugarcane in the world, producing through it more than 37 million tons of sugar and 27 billion liters of ethanol. Currently there is an increase in the demand for fuel ethanol consumption, especially due to the variation of the price of petroeum in the international market and the renewable character of this energy source. The aim of this work is to increase the ethanol production from the re-sizing of the fermentation cooling system of an industrial plant. In order, it was necessary to perform an energy balance of the fermentation cooling system, in its old configuration, and to redistribute the plates to meet the need for heat absorption of the first and second stages. Another important point was the expansion of the second stage, increasing the residence
\end{abstract}

time of the fermented wort. The implemented modifications generated an increase in plant productivity measured by the amount of sugarcane destined for ethanol production, which doubled in relation to the previous harvest, the quantity of wine produced that was $36 \%$ higher, the increase of $0.9 \%$ in quantity of total reducing sugars (ART) present in the food, which increased by $6 \%$ the alcoholic content of the wine, giving a $52 \%$ higher production in the studied period, maintaining the fermentation temperature in the optimum range of yeast. These results evidenced the increase in the productivity of the system and also provided a reduction in the cost of maintenance by reducing the number of heat exchangers and pumps in operation in the plant.

KEYWORDS: Energy balance, Alcoholic fermentation, Plate heat exchangers 


\section{INTRODUÇÃO}

O Brasil ocupa uma posição de destaque em relação a produção de cana-de-açúcar, ocupando a primeira posição no mundo com cerca de 633,26 milhões de toneladas colhidas na safra 2017/2018, número ainda 3,6\% inferior ao da safra 2016/2017. A queda na colheita ocorreu devido à diminuição da área plantada nesta safra com 8,73 milhões de hectares contra 9,05 milhões de hectares da safra 2016/2017, número 4,6\% maior que na safra anterior 2015/2016, estando a cana-de-açúcar atrás apenas da soja e do milho, em relação à área plantada no país (CONAB, 2018).

A produção de açúcar na safra 2018/2019 recuou em relação à safra anterior para 37,87 milhões de toneladas, queda de $2,1 \%$. O principal propulsor foi o baixo preço do produto no mercado interno e externo. $O$ etanol, por sua vez, apresentou uma melhor remuneração frente ao açúcar o que proporcionou praticamente a manutenção do mesmo volume de produção, 27,76 bilhões de litros, apenas $0,2 \%$ de queda em relação à safra anterior, mesmo com a redução da área plantada devido à um direcionamento maior da moagem para a produção de álcool (CONAB, 2018).

O etanol anidro apresentou leve alta, cerca de 0,1\% ante a safra 2016/2017, enquanto que o etanol hidratado apresentou queda de $0,4 \%$. Entretanto, no início da safra a expectativa era de uma queda ainda maior na produção do álcool hidratado, cerca de 10,7\%. A partir de outubro de 2017 houve maior consumo do etanol hidratado e, consequentemente, sua produção, tendência esta que permanecerá para a safra 2018/2019 com estimativa de crescimento de 11,6\%, totalizando 30,41 bilhões de litros (CONAB, 2018), o que requer maior atenção as unidades produtoras de etanol.

Diante da necessidade das indústrias em direcionar seu mix para a produção de etanol, tornou-se imprescindível o estudo e desenvolvimento de projetos que objetivassem a maximização da produtividade de etanol, ao menor custo possível, aproveitando a estrutura já existente, tendo em vista o cenário de crise existente no setor sucroenergético (Manoel et al., 2017).

Entre as opções para a elevação de produtividade do setor de fabricação de etanol, estão a aquisição de novos equipamentos, repotenciamento dos equipamentos já existentes ou redimensionamento de etapas dos processos. Uma das formas mais econômicas de aumentar a capacidade de produção do setor de fabricação de etanol é redimensionar o processo, utilizando equipamentos já existentes na planta industrial, apenas os realocando ou reorganizando a fim de que atendam melhor às necessidades operacionais e proporcionem a elevação da produtividade.

Nesse sentido, um dos principais passos a serem executados para elevar a produção de etanol é interferir adequadamente no processo de fermentação alcoólica ou no processo de destilação, que são as duas etapas mais importantes na produção do etanol.

No caso específico da etapa de fermentação alcoólica, trabalhar na eficiência da refrigeração das dornas de fermentação pode ser uma boa alternativa, pois é possível aumentar 
a quantidade de matéria-prima a ser processada sem superaquecer o sistema, o que não provocaria redução da sua eficiência e assim, aumentaria a produção etílica.

Sendo assim, o principal objetivo deste trabalho foi aumentar a produção de etanol a partir do redimensionamento do sistema de resfriamento da fermentação de uma planta industrial localizada no estado de Pernambuco.

\section{REVISÃO BIBLIOGRÁFICA}

Trocadores de calor de placas

Trocadores de calor de placas vêm sendo utilizados nas indústrias com mais intensidade desde a década de 1930 e, ao longo do tempo, foi ganhando espaço até em operações onde os trocadores casco-tubo predominavam. Hoje, os trocadores de placas são amplamente utilizados em operações líquido-líquido, que ocorram a temperaturas e pressões intermediárias (abaixo de 250 ㅇ C e 25 bar) devido a sua flexibilidade operacional, alta eficiência, economia de espaço e facilidade de limpeza (Hewitt et al., 1994) (Dryden, 2018).

As desvantagens apresentadas são o uso de gaxeta para separação dos fluídos, o que não permite a operação em pressões e temperaturas mais elevadas, alta perda de carga provocada por apresentar ranhuras muito estreitas. O fato da área das ranhuras serem reduzidas não trazem prejuízos, pois gera maior turbulência elevando, por consequência, o coeficiente convectivo de transferência de calor (Gut, 2003).

Os trocadores de calor de placas podem trabalhar com o fluxo em série ou em paralelo, a depender da distribuição dos fluidos quente e frio e a disposição das gaxetas (Figura 1), perfuração das placas (Figura 2) e localização dos bocais de entrada e saída do trocador (Figura 3) (Gut, 2003).

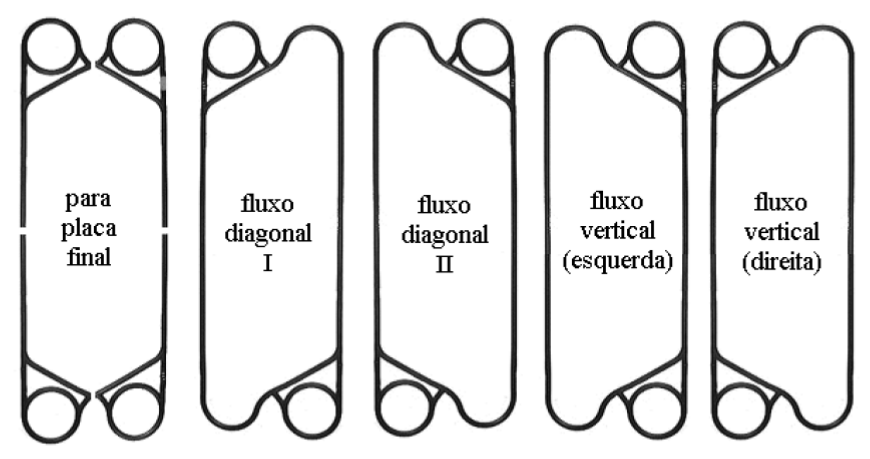

Figura 1: Possíveis posições de uma gaxeta 


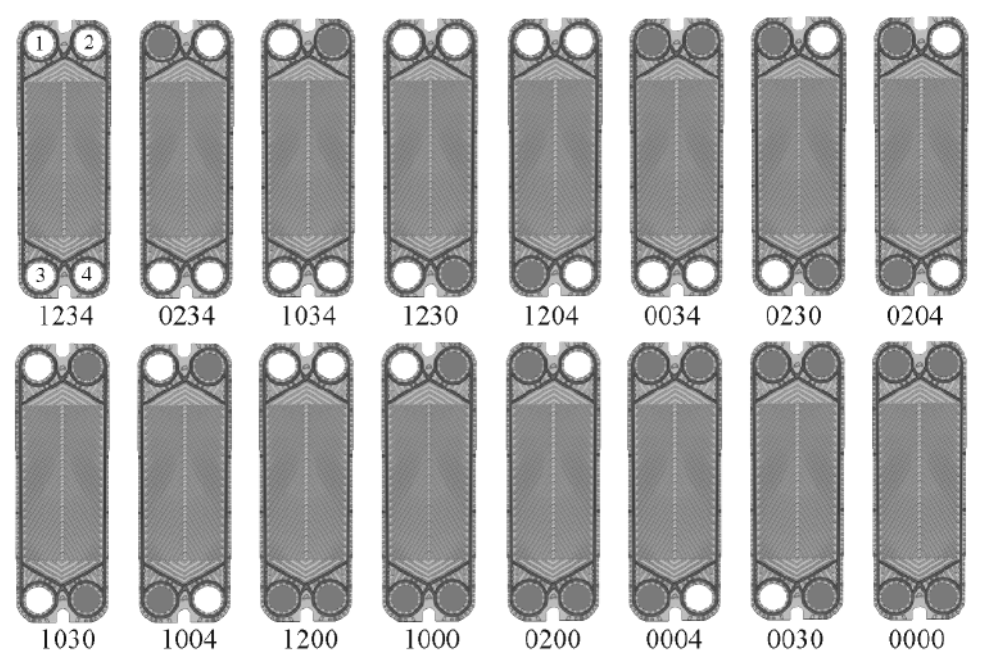

Figura 2: Posições de perfuração de placas possíveis

fluxo diagonal

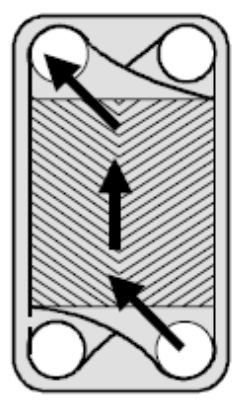

ascendente

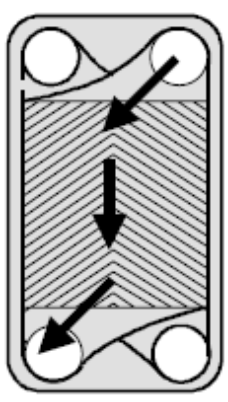

descendente

fluxo vertical

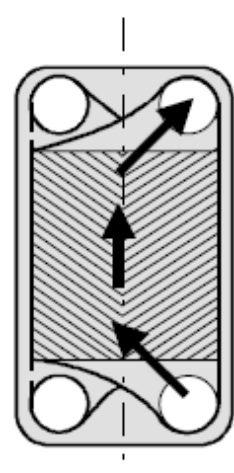

ascendente (direita)

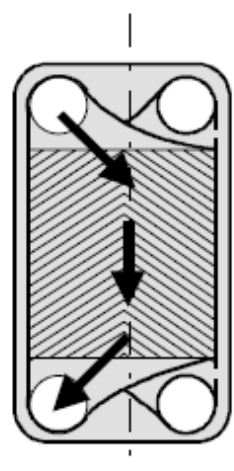

descendente (esquerda)

Figura 3: Tipos de fluxo nos canais de escoamento

Estes trocadores são constituídos de um conjunto de placas de aço inoxidável com ranhuras, agrupadas de acordo com o posicionamento das gaxetas para o correto direcionamento dos fluidos quente e frio e comprimidas através do aperto dos parafusos que prende a placa móvel a fixa como apresentado na Figura 4. 

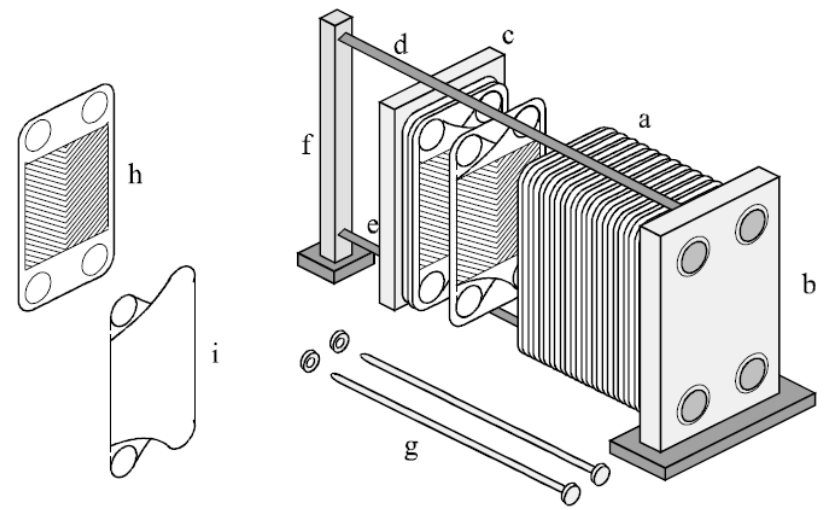

a. Pacote de placas aberto

b. Placa fixa com conexões

c. Placa de aperto móvel

d. Barramento superior

e. Barramento inferior

f. Coluna de sustentação

g. Parafusos de aperto

h. Placa corrugada chevron

i. Gaxeta

Figura 4: Componentes de um trocador de calor de placas gaxetado

A placa tipo Chevron ou espinha de peixe é a mais utilizada normalmente, o detalhamento da mesma é apresentado na Figura 5.

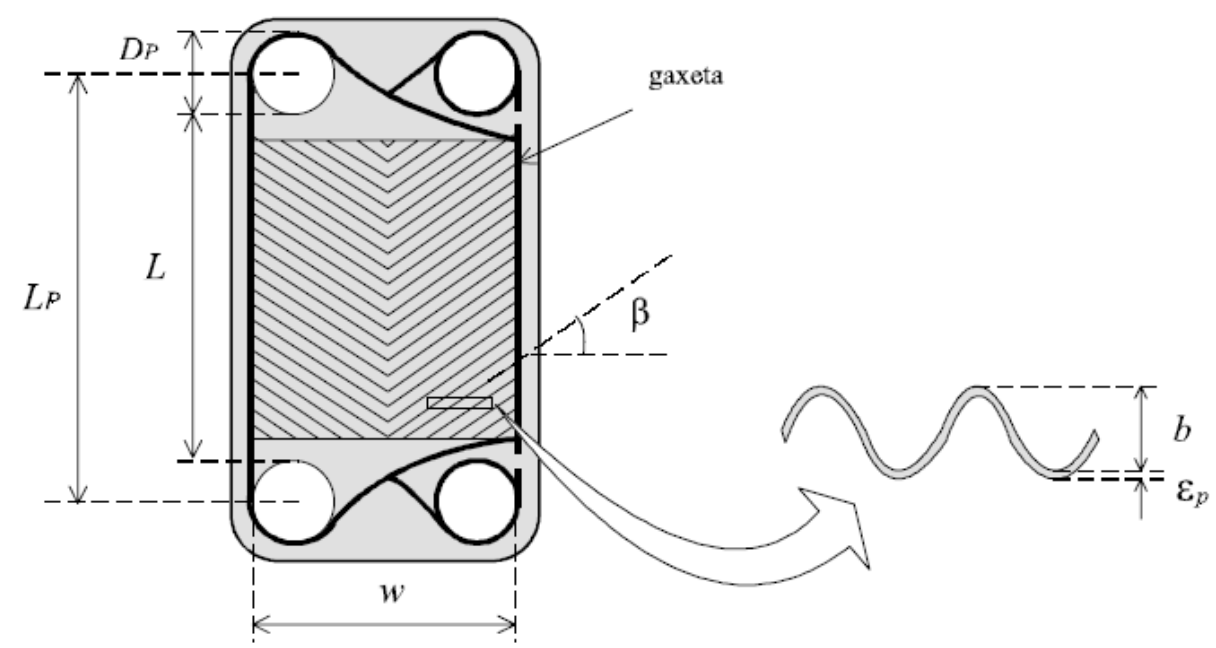

Figura 5: Placa tipo Chevron

Em que: Lp: Comprimento efetivo de escoamento $(\mathrm{mm})$; Dp: Diâmetro do bocal $(\mathrm{mm})$; L: Comprimento efetivo para troca térmica $(\mathrm{mm})$; w: Largura efetiva do canal $(\mathrm{mm}) ; \beta$ : Ângulo de inclinação das ranhuras; b: Espessura média da ranhura $(\mathrm{mm}) ; \varepsilon \_p$ : Espessura da placa $(\mathrm{mm})$.

Para dimensionar um trocador de calor de placas, duas considerações são importantes para o cálculo da área de transferência de calor. A primeira delas é que nas placas de fechamento, primeira e última placas não há troca térmica, ou seja, considera-se para efeito de cálculo que o número de placas térmicas (Npt) é o número de placas totais ( $\mathrm{Np}$ ) menos as de fechamento (Equação 1) (Incropera e Dewitt, 2008).

$\mathrm{Npt}=\mathrm{Np}-2$ 
A segunda consideração é que pelo fato de haver ranhuras a área efetiva da placa, Ap, é maior que a área estrutural. O fator de correção $(\Phi)$ da área varia entre 1,15 e 1,25 o que pode ser descrito pela Equação 2 (Kakaç et al., Liu, 2012).

$$
A p=\Phi w L
$$

Para um equacionamento preciso de um trocador de placas, além de conhecer suas dimensões, é importante conhecer as configurações do equipamento, tais como o número de passes, número de canais, se o fluxo é em contracorrente ou cocorrente, onde é a alimentação e a saída, se o fluxo é diagonal ou vertical.

\section{METODOLOGIA}

Os experimentos foram planejados para uma planta industrial localizada no estado de Pernambuco e baseou-se nos mecanismos de transferência de calor para redimensionar o sistema de refrigeração das dornas de fermentação para que fosse possível receber uma quantidade maior de matéria prima sem superaquecer o sistema, o que reduziria sua eficiência.

A liberação de energia durante a fermentação é da ordem de 138,89 kcal para cada quilograma de Açúcares Redutores Totais (ART) fornecido ao sistema, portanto, para aumentar a produção etanol sem perda de eficiência pela levedura, torna-se evidente a necessidade de aumentar a capacidade da planta de absorver calor (Leão e Amorin, 2005).

A planta original de fermentação selecionada para redimensionamento do sistema de resfriamento cuja configuração está apresentada na Figura 6 é composta por dez dornas, cada uma com capacidade para 200.000 L. A alimentação do mosto e do fermento tratado acontece em quatro dornas (dornas 1, 2, 3 e 4, Figura 6) que estão interligadas entre si formando o primeiro estágio de fermentação, onde a maior parte da conversão dos açúcares em álcool acontece e, consequentemente, onde é liberada a maior quantidade de energia.

A antiga configuração da planta apresenta, após o primeiro estágio, duas linhas de fermentação, ambas compostas por três dornas, o segundo (dornas 5 e 10), o terceiro (dornas 6 e 9), e o quarto (dornas 7 e 8) estágio (Figura 6). A existência das duas linhas permite que a limpeza das dornas seja realizada com a planta em operação.

Cada dorna é refrigerada individualmente. O mosto fermentado é succionado do fundo da dorna para um trocador de calor de placas que opera transferindo calor do mosto para água proveniente da torre de resfriamento. O retorno do mosto à dorna ocorre na parte inferior do equipamento, mantendo a região sob constante agitação evitando a decantação do fermento e, consequente, perda de eficiência na conversão de substrato em produto.

Os trocadores instalados na fermentação são todos do fabricante APV, dos quais cinco são do modelo R56 e cinco do modelo R88, conforme disposição apresentada na Figura 6. 


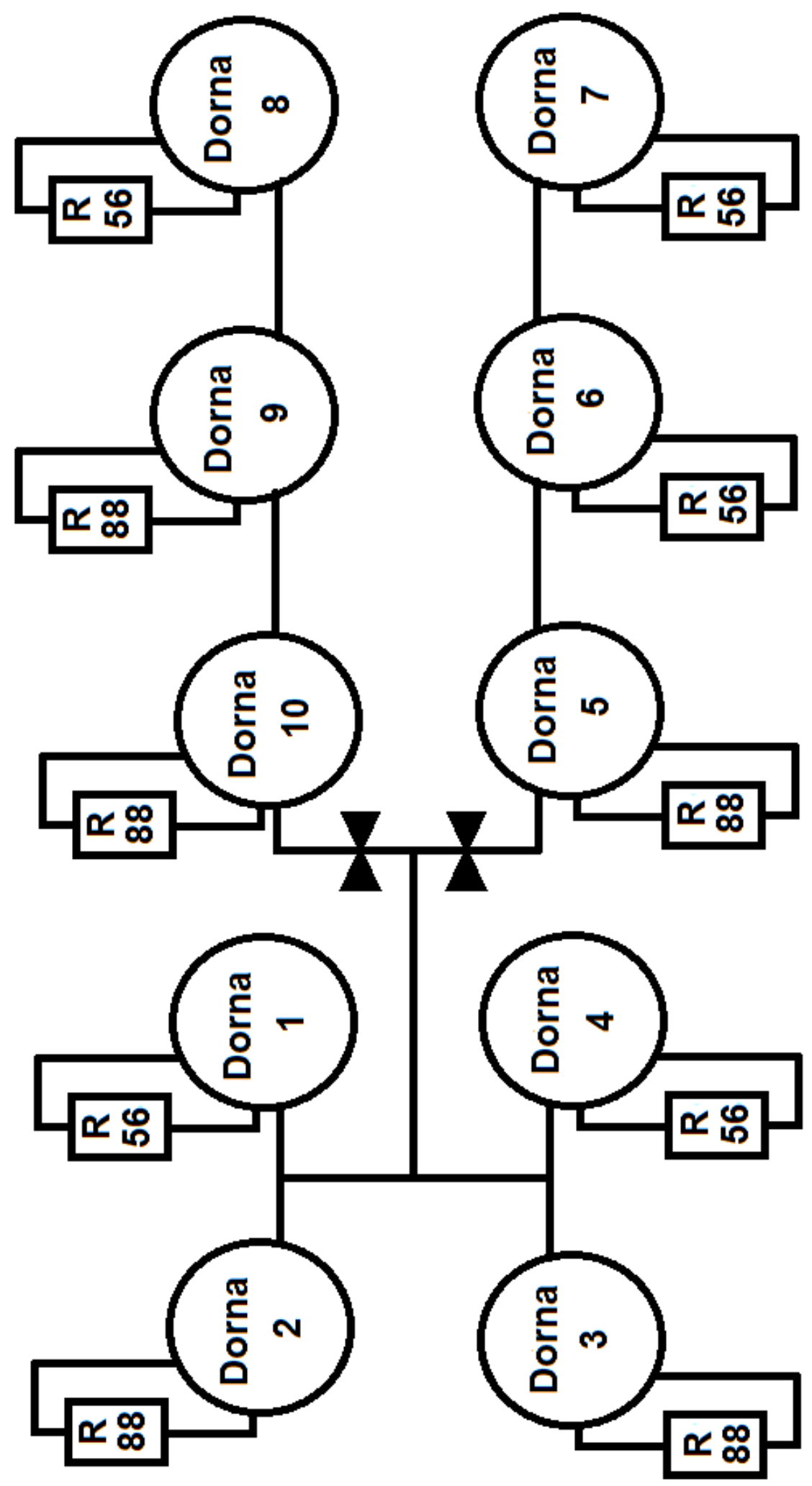

Figura 6: Configuração da planta de fermentação antiga. 
A Tabela 1 apresenta a capacidade de resfriamento (CRA) da planta de fermentação antiga.

Tabela 1 - Capacidade de resfriamento da planta de fermentação antiga

\begin{tabular}{|c|c|c|c|}
\hline Dorna & $\begin{array}{l}\text { Modelo do trocador } \\
\text { de calor }\end{array}$ & № de placas & Área efetiva - $m^{2}$ \\
\hline \multicolumn{4}{|c|}{ Primeiro estágio } \\
\hline 1 & R56 & 68 & 36,3 \\
\hline 2 & R88 & 76 & 54,0 \\
\hline 3 & $\mathrm{R} 88$ & 78 & 55,5 \\
\hline 4 & R56 & 68 & 36,3 \\
\hline \multicolumn{4}{|c|}{ Segundo estágio } \\
\hline 5 & R88 & 78 & 41,8 \\
\hline 10 & R88 & 78 & 55,5 \\
\hline \multicolumn{4}{|c|}{ Terceiro estágio } \\
\hline 6 & R56 & 74 & 39,6 \\
\hline 9 & R88 & 78 & 55,5 \\
\hline \multicolumn{4}{|c|}{ Quarto estágio } \\
\hline 7 & R56 & 78 & 41,8 \\
\hline 8 & R56 & 76 & 40,7 \\
\hline
\end{tabular}

Para calcular a área necessária para realizar a troca térmica considerou-se o fluxo de calor através de uma placa plana com largura $y$, comprimento $z$ e espessura $x$. Considerando estado estacionário o fluxo de calor na espessura da placa tem-se:

$\frac{d^{2} T}{d x^{2}}=0$

Nessas condições a Equação 3 tem como solução:

$T=A+B x$

Em que:

$\left.T\right|_{x=0}=T_{i} \quad$ (temperatura inicial)

$\left.T\right|_{x=L}=T_{f} \quad$ (temperatura final) 
Portanto, quando $x=0$ obtém-se a Equação 6:

$A=T_{i}$

Logo:

$B=\frac{T_{f}-T_{i}}{L}$

Substituindo $A$ e $B$ na Equação 4:

$T=T_{i}+\frac{T_{f}-T_{i}}{L} x$

O fluxo de calor por condução na direção x é representado pela equação 9:

$q_{x}=-k \frac{d T}{d x}=-k \frac{T_{f}-T_{i}}{L}=k \frac{T_{i}-T_{f}}{L}$

Pode-se representar a Equação 9 da seguinte forma:

$$
q_{x}=\frac{k}{L} \Delta T=u \Delta T
$$

Em que: $u$ é a resistência a transferência de calor por condução e a variação de temperatura é a força motriz da transferência de calor. A taxa de transferência de calor pode ser obtida integrando duplamente a Equação 11.

$$
\begin{aligned}
& Q=\int_{0}^{y} \int_{0}^{Z} q d y d z \\
& Q=q Y Z \\
& Q=k \frac{T_{i}-T_{f}}{L} Y Z \\
& Q=\frac{k}{L} \Delta T Y Z \\
& Q=u \Delta T Y Z
\end{aligned}
$$

Em que: $Y$ multiplicado por $Z$ representa a área efetiva da placa (Equação 16):

$$
Q=u A \Delta T
$$

Como o trocador de placas em estudo opera trocando energia entre fluidos, deve-se considerar também o fluxo de calor por convecção em ambos os lados da placa. Para tal, adotase o conceito de resistência ao fluxo de calor. 


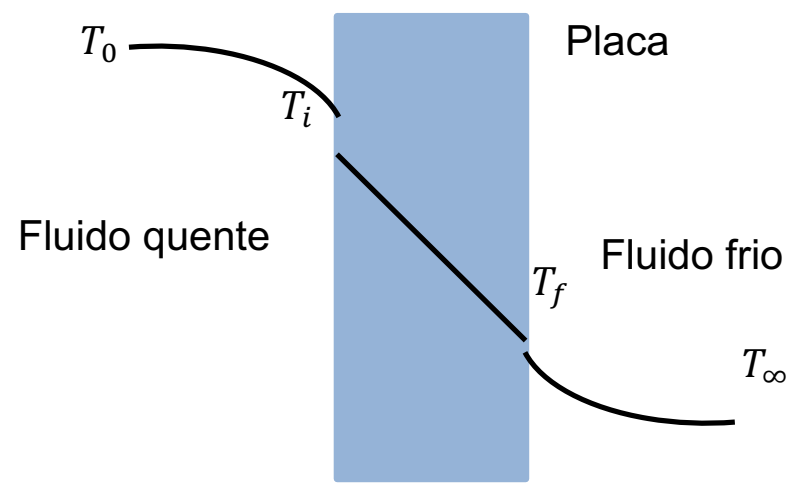

Figura 7: Representação do fluxo de calor através de uma placa

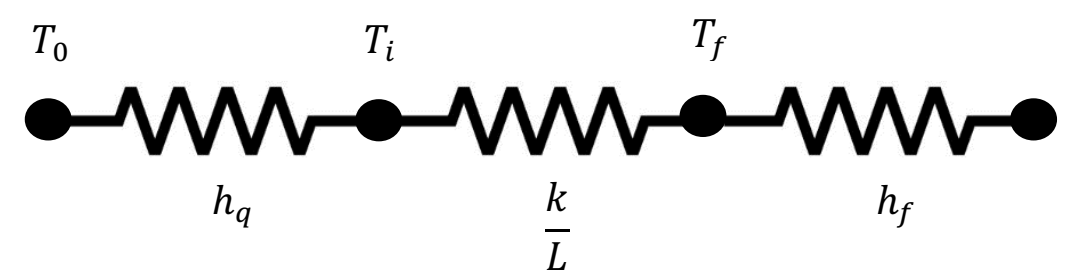

Figura 8: Representação das resistências à transferência de calor

Com isso, para o sistema estudado o coeficiente de resistência a transferência de calor global é a soma das três resistências.

$$
u=h_{q}+\frac{k}{L}+h_{f}
$$

Desta forma, foi proposto o aumento do tempo de residência e da área de troca térmica disponível no segundo estágio, e aumento da superfície de troca térmica também no primeiro estágio, onde, aproximadamente 70 a $80 \%$ da conversão de ART em etanol acontece. A área dos trocadores dos estágios finais também foram recalculados.

Para acompanhar a efetividade das modificações realizadas foi monitorado a temperatura das dornas, o percentual de ART fornecido ao sistema, a quantidade de vinho produzida e o teor alcoólico do vinho a ser destilado. Todos esses dados foram comparados ao monitoramento realizado na safra anterior (2017/2018) para demostrar a evolução.

A determinação do ART no mosto de alimentação do primeiro estágio foi realizada a partir do método Eynon-Lane, que se baseia na redução de ions $\mathrm{Cu}^{2+}$ da solução Feeling $\mathrm{A}$. A metodologia foi adaptada de (Caldas, 1998).

Preparo do titulante:

- Transferir $10 \mathrm{~mL}$ da amostra para um béquer de $250 \mathrm{~mL}$;

- Acrescentar $200 \mathrm{~mL}$ de água destilada;

- Adicionar $10 \mathrm{~mL}$ de $\mathrm{HCl}$ a 50\%;

- Levar ao Banho Maria, à $70{ }^{\circ} \mathrm{C}$ por 15 minutos;

- Resfriar a amostra até temperatura ambiente; 
- Neutralizar com $\mathrm{NaOH}$ a 20\%;

- Transferir para balão volumétrico de $500 \mathrm{~mL}$ e completar com água destilada.

Preparo da solução a ser titulada:

- Adicionar 2,5 mL de solução Feeling A;

- Adicionar 2,5 mL de solução Feeling B;

- Acrescentar $40 \mathrm{~mL}$ de água destilada;

- Aguardar a mistura entrar em ebulição;

- Inserir 3 gotas do indicador azul de metileno;

- Titular até mudar da cor azul para vermelho tijolo.

O valor de ART no mosto de alimentação é obtido dividindo-se 250 pelo volume gasto na bureta.

Para determinar o ART, após o primeiro estágio fermentativo e o residual no vinho, é importante centrifugar a amostra para evitar que a fermentação continue acontecendo, enquanto a amostra não é analisada. Para maior segurança, a amostra deve ser refrigerada até $10{ }^{\circ} \mathrm{C}$, pois caso ainda haja um resíduo de fermento a temperatura baixa desacelerará seu metabolismo minimizando os erros de análises.

Os equipamentos utilizados para estas análises foram: Centrifuga Excelsa II modelo 206 BL, Banho Maria Solab modelo 150/10, pHmetro Digimed modelo DM-20 e aparelho Redutec

Outro parâmetro de grande relevância para mensurar a eficácia das modificações propostas neste trabalho é o acompanhamento do teor alcoólico ou grau Gay-Lussac $\left({ }^{\circ} \mathrm{GL}\right)$ do vinho produzido, sendo sua análise realizada a cada duas horas. Assim como as outras análises, a comparação entre a safra atual e a anterior também foi realizada. A metodologia de análise é exposta abaixo.

- Adicionar $25 \mathrm{~mL}$ de amostra ao microdestilador;

- Coletar destilado em balão de $50 \mathrm{~mL}$;

- Realizar leitura no densímetro.

O resultado da leitura é multiplicado por 2 , desta forma, é obtido o teor alcoólico $\left({ }^{\circ} \mathrm{GL}\right)$ do vinho. Os equipamentos utilizados nesta análise foram o Microdestilador TECNAL modelo TE-012 e o Densímetro Automático Rudoph modelo DDM 2911.

\section{RESULTADOS E DISCUSSÃO}

Após uma avaliação detalhada da planta de fermentação original (Figura 6) foi identificado que os trocadores de calor (modelo R56), acoplados às dornas 6 e 7 (do terceiro e quarto estágio, respectivamente), estavam superdimensionados, portanto remanejaram-se parte das placas destes trocadores para os trocadores (modelo R56) acoplados às dornas 1 e 4, do primeiro estágio e ao trocador (modelo R56) acoplado à dorna 8, do quarto estágio.

Sabendo que as dornas 6 e 9 (terceiro estágio) e 7 e 8 (quarto estágio) (Figura 6) não operam ao mesmo tempo, flexibilizou-se o sistema de resfriamento para que apenas um trocador de calor atendesse o par de dornas desses estágios. Sendo assim, estas dornas ficaram operando apenas com um trocador de calor, e não com dois como inicialmente.

A partir da análise anterior, foi apresentada uma nova configuração para a planta de fermentação, como mostra a Figura 9. 


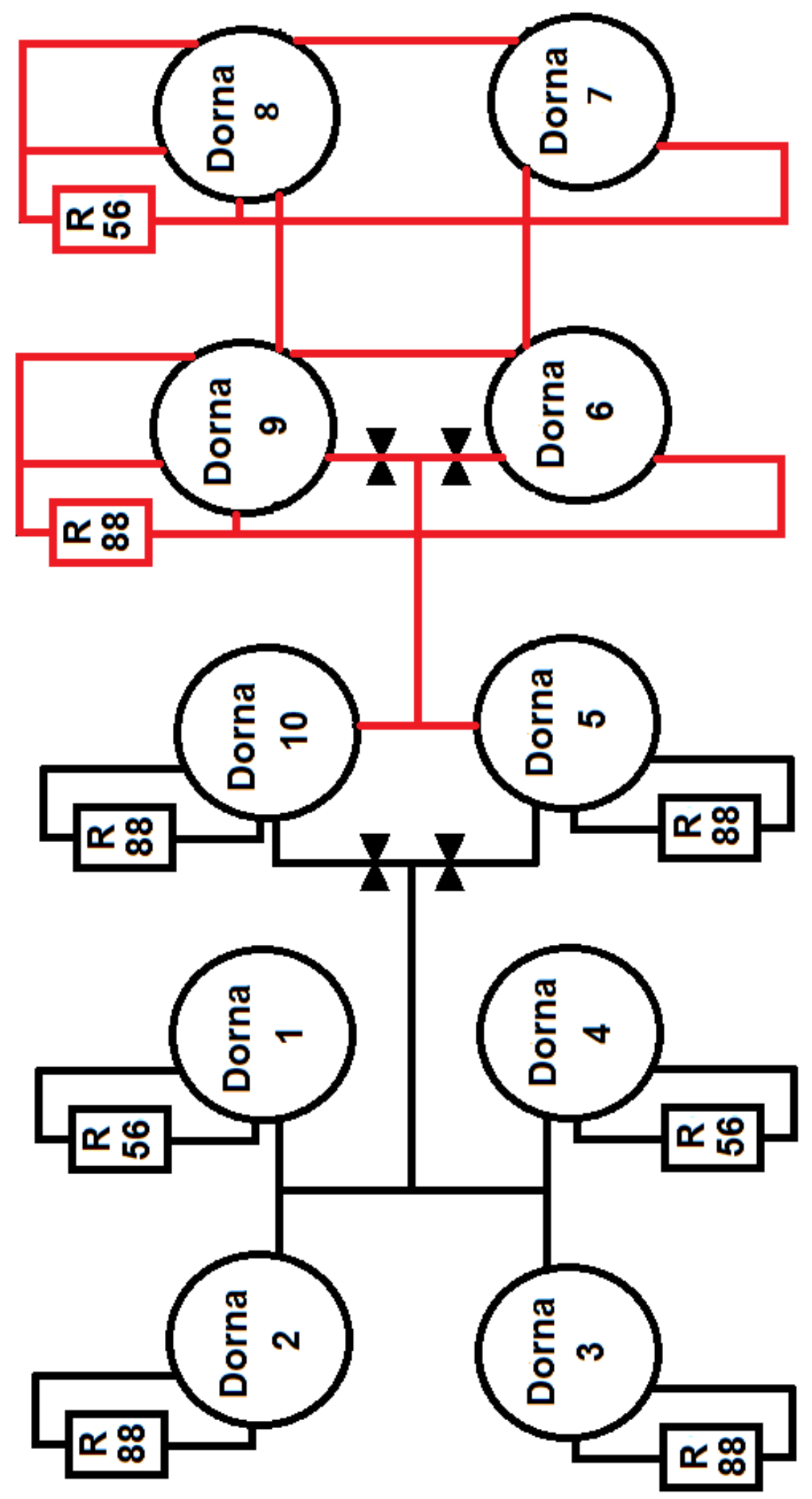

Figura 9: Nova configuração da planta de fermentação 
A Tabela 2 apresenta as modificações quantitativas relacionadas com a capacidade de refrigeração da planta de fermentação.

Analisando a Tabela 2, observa-se que os trocadores de calor do modelo R56 do primeiro estágio (Figura 9), acoplados às dornas 1 e 4, tiveram cada um deles um aumento de 34 placas, o que elevou sua área efetiva de transferência de calor de 36,3 para $51 \mathrm{~m}^{2}$. Similarmente, o trocador de calor, modelo R56 acoplado à dorna 8, quarto estágio, houve um aumento de 14 placas o que produz uma elevação na sua área efetiva de transferência de calor de 40,7 para 45 $\mathrm{m}^{2}$.

Tabela 2. Capacidade de resfriamento da planta de fermentação antiga (CRA) e a proposta (CRP)

\begin{tabular}{|c|c|c|c|c|c|}
\hline Dorna & $\begin{array}{c}\text { Modelo do } \\
\text { trocador de } \\
\text { calor }\end{array}$ & $\begin{array}{c}\text { № de placas } \\
\text { (CRA) }\end{array}$ & $\begin{array}{c}\text { Área efetiva } \\
-\mathrm{m}^{2} \\
\text { (CRA) }\end{array}$ & $\begin{array}{c}\text { № de placas } \\
\text { (CRP) }\end{array}$ & $\begin{array}{c}\text { Área efetiva } \\
-\mathrm{m}^{2} \\
\text { (CRP) }\end{array}$ \\
\hline \multicolumn{6}{|c|}{ Primeiro estágio } \\
\hline 1 & R56 & 68 & 36,3 & 102 & 51,0 \\
\hline 2 & R88 & 76 & 54,0 & 76 & 54,0 \\
\hline 3 & R88 & 78 & 55,5 & 78 & 55,5 \\
\hline 4 & R56 & 68 & 36,3 & 102 & 51,0 \\
\hline \multicolumn{6}{|c|}{ Segundo estágio } \\
\hline 5 & R88 & 78 & 55,5 & 78 & 55,5 \\
\hline 10 & $\mathrm{R} 88$ & 78 & 55,5 & 78 & 55,5 \\
\hline \multicolumn{6}{|c|}{ Terceiro estágio } \\
\hline 6 & R56 & 74 & 39,6 & - & - \\
\hline 9 & $\mathrm{R} 88$ & 78 & 55,5 & 78 & 55,5 \\
\hline \multicolumn{6}{|c|}{ Quarto estágio } \\
\hline 7 & R56 & 78 & 41,8 & - & - \\
\hline 8 & R56 & 76 & 40,7 & 90 & 45,0 \\
\hline
\end{tabular}

Com a retirada das placas dos trocadores (modelo R56) acoplados às dornas 6 e 7 (Quadro 2, Figura 9), foi possível reduzir os custos de manutenção e ter uma economia de energia na planta de fermentação proposta. Isto foi possível, porque estes trocadores de calor (das dornas 6 e 7), assim como as bombas associadas a eles foram desativados totalmente. Além disso, após o redimensionamento, sobraram 70 placas do trocador modelo R56, que agora estão disponíveis como reserva técnica para utilização caso haja problemas de trincas, furos e vedação nas placas em operação na forma de manutenção corretiva.

Além da nova configuração da planta de fermentação promover uma redução nos custos de manutenção e uma economia de energia, a configuração proposta, promoveu, também um aumento na produtividade da fermentação como é apresentado pela comparação dos dados referentes à produção do início da safra 2017/2018 - 21 de junho de 2017 - e da safra atual - 18 de agosto de 2018 - até o dia 31 de outubro das respectivas safras.

$O$ redimensionamento do sistema de resfriamento das dornas de fermentação proposto ampliou a capacidade de absorção de calor de $2314 \mathrm{Mcal} / \mathrm{h}$ para $2688 \mathrm{Mcal} / \mathrm{h}$, portanto, tornouse possível receber uma quantidade maior de matéria-prima sem que a temperatura ultrapassasse os $35^{\circ} \mathrm{C},\left(25-35^{\circ} \mathrm{C}\right)$, faixa ótima de temperatura para fermentação em estudo (Souza, et al., 2018; Techaparin, et al, 2017; Wang, et al., 2014; Zabed, et al., 2009) como pode ser observado na Tabela 3. 
Os cálculos do redimensionamento foram realizados estimando como $35{ }^{\circ} \mathrm{C}$ a temperatura de entrada no trocador e $31{ }^{\circ} \mathrm{C}$ a temperatura na saída, e uma vazão de entrada de matéria-prima no sistema $30 \%$ maior que na safra anterior. A elevação de temperatura em relação à safra anterior (2017/2018) foi baixa, menor que 6\%, e se deve a elevação da vazão de alimentação, estando dentro do limite máximo aceitável de $35{ }^{\circ} \mathrm{C}$ e dentro das condições de projeto o que demonstra e eficácia das medidas adotadas.

A Figura 10 indica a quantidade de cana destinada à produção de etanol, que no período estudado foi duas vezes e meia superior à safra anterior (2017/2018), demonstrando que há de fato um direcionamento para produção de álcool por razões comerciais como projetado pela CONAB (2018). Os dias em que os gráficos se aproximam de zero, são aqueles em que a planta parou para realizar manutenção.

Tabela 3 - Comparativo entre as temperaturas médias das dornas de fermentação na safra atual e safra anterior

\begin{tabular}{|c|c|c|c|}
\hline Dorna & $\begin{array}{c}\text { Temperatura da } \\
\text { dorna }\left({ }^{\circ} \mathrm{C}\right) \\
\text { Safra } 2017 / 2018\end{array}$ & $\begin{array}{c}\text { Temperatura da } \\
\text { dorna }\left({ }^{\circ} \mathrm{C}\right) \\
\text { Safra } 2018 / 2019\end{array}$ & $\begin{array}{c}\text { Elevação da } \\
\text { temperatura (\%) }\end{array}$ \\
\hline \multicolumn{4}{|c|}{ Primeiro estágio } \\
\hline 1 & 31,68 & 32,12 & 1,39 \\
\hline 2 & 31,95 & 33,53 & 4,95 \\
\hline 3 & 31,86 & 32,12 & 0,82 \\
\hline 4 & 31,83 & 33,40 & 4,93 \\
\hline \multicolumn{4}{|c|}{ Segundo estágio } \\
\hline 5 & 31,39 & 32,75 & 4,33 \\
\hline 10 & - & 33,27 & - \\
\hline \multicolumn{4}{|c|}{ Terceiro estágio } \\
\hline 6 & 31,40 & 33,13 & 5,51 \\
\hline 9 & - & - & - \\
\hline \multicolumn{4}{|c|}{ Quarto estágio } \\
\hline 7 & 31,21 & 32,43 & 3,91 \\
\hline 8 & 31,16 & - & - \\
\hline
\end{tabular}

Estes dados estão diretamente em consonância com o principal objetivo deste trabalho, que foi aumentar a produção de etanol através da ampliação da capacidade de processamento de matéria prima na planta de produção de etanol da Usina. 


\section{CANA MOÍDA PARA PRODUÇÃO DE ETANOL}

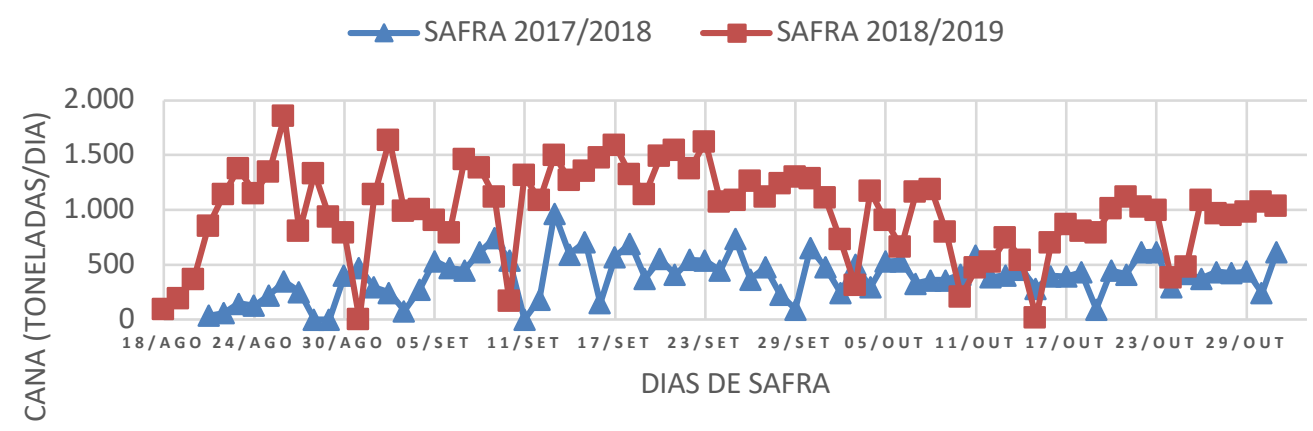

Figura 10: Comparativo da quantidade de cana-de-açúcar moída para produção de etanol

Outro dado relevante, que mostra que a planta de fermentação recebeu uma quantidade maior de matéria prima, é a quantidade de vinho destilada produzida por dia (Figura 11). Observa-se, nesta figura, que a produção de vinho destilado por dia é maior durante quase todo o período da safra 2018/2019, quando comparado ao da safra $2017 / 2018$. A produção do vinho destilado foi $36,55 \%$ maior na safra atual (2018/2019), o que indica um aumento real na capacidade de produção da planta de fermentação com a configuração proposta neste trabalho.

Para este cálculo foi considerado que toda levedura adicionada na fermentação é removida durante a centrifugação, o que é apenas uma aproximação, tendo em vista que há perda de fermento na etapa de centrifugação do mosto, podendo aproximar o volume de vinho ao volume de mosto.

\section{VOLUME DE VINHO DESTILADO POR DIA}

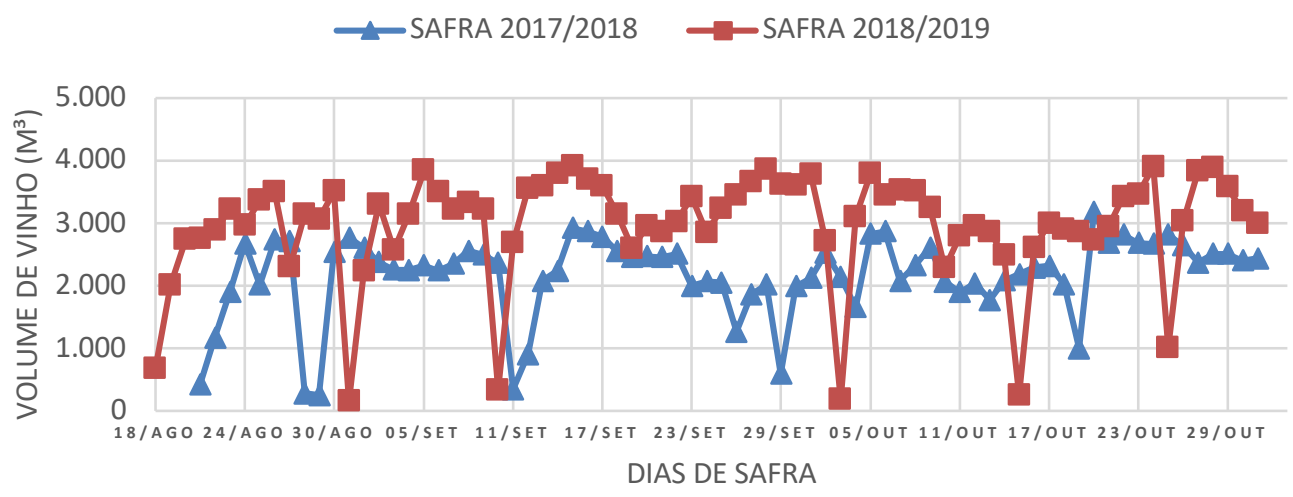

Figura 11: Comparativo da quantidade de vinho destilado

Outro ponto a ser ressaltado é que, além de uma quantidade de mosto maior, a quantidade de açúcares redutores totais (ART) presentes na vazão de alimentação cresceu de $12,62 \%$ para $13,53 \%$, contribuindo para a geração de calor nas dornas de fermentação (Figura 12). 


\section{ART NA ALIMENTAÇÃO DA FERMENTAÇÃO}

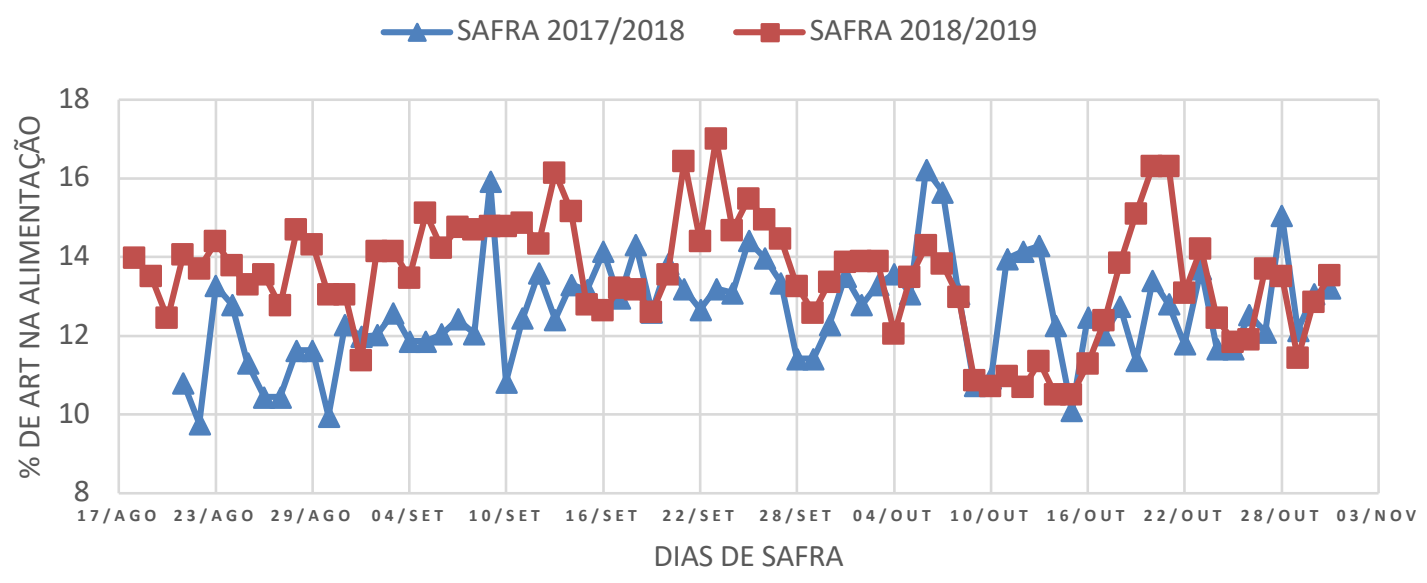

Figura 12: Comparativo do percentual de ART presente na alimentação

Para a elevação do ART, foi necessário elevar o consumo de melaço, pois o mesmo apresenta ART médio de $53,72 \%$ em sua composição contra $10,63 \%$ do caldo. O fato é evidenciado pelo estoque de mel (Figura 13), que caiu de 571 toneladas em média na safra anterior para 360 toneladas nesta safra. Trabalhar com estoques mais baixos de melaço favorece também a eficiência industrial, pois a estocagem deste produto a temperaturas altas e por um período prolongado pode acarretar na degradação dos açúcares presentes no mel (Rein, 2008).

\section{ESTOQUE DE MEL}

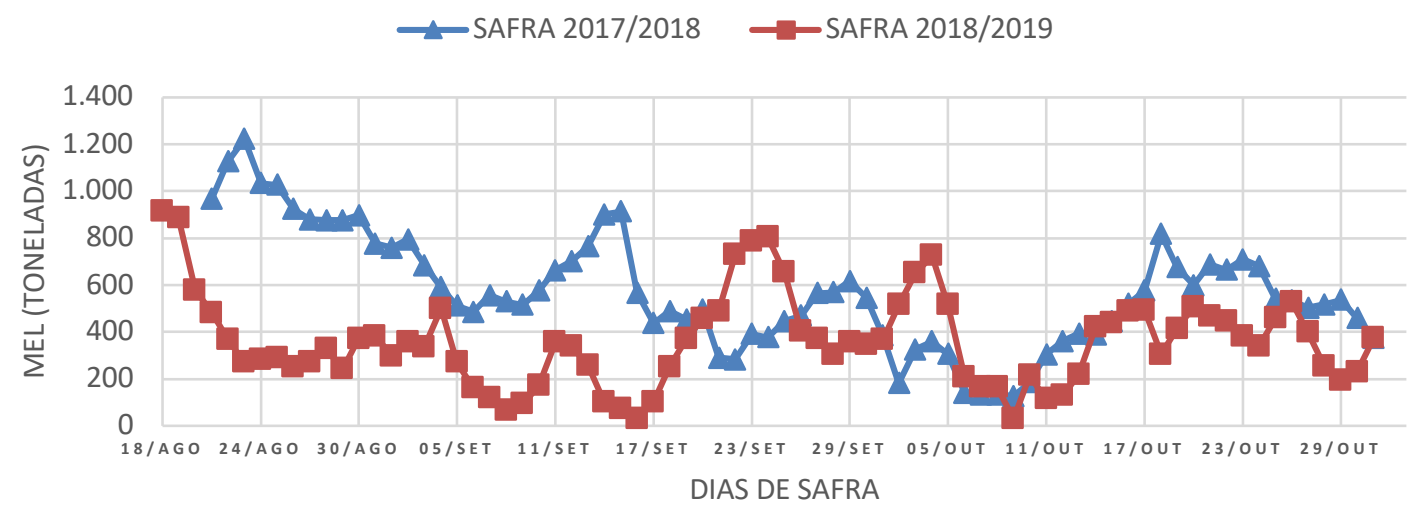

Figura 13: Comparativo do estoque de mel disponível

Operar com o ART mais alto na fermentação viabiliza a elevação do percentual de etanol no vinho a ser destilado, o que eleva a produtividade do sistema de destilação e diminui o consumo de vapor da planta. $\mathrm{O}^{\circ} \mathrm{GL}$ da safra atual é $6 \%$ superior ao da safra anterior, como pode ser observado na Figura 14. 


\section{PERCENTUAL DE ETANOL NO VINHO}

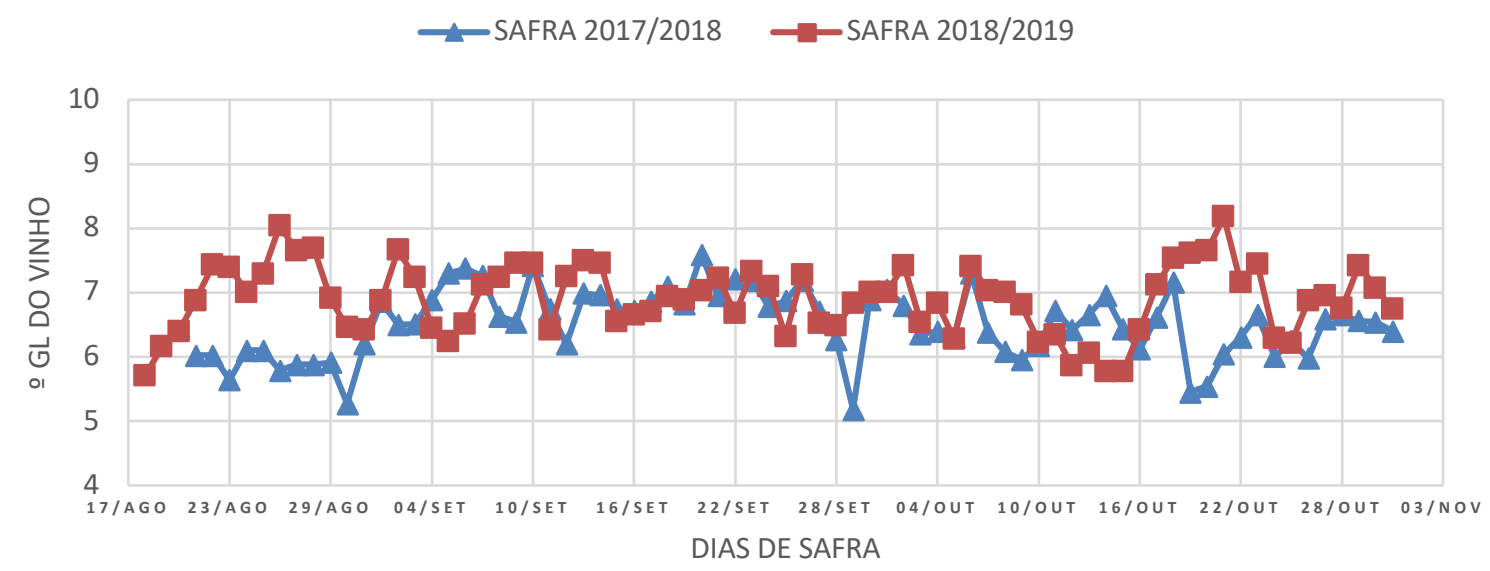

Figura 14: Comparativo do 9 GL do vinho produzido

As modificações realizadas na planta de fermentação possibilitaram aumentar, significativamente, a produção de etanol da safra atual quando comparado com a safra passada (Figura 15). Na safra atual, foram produzidos 15.729.985 L de etanol em 75 dias de safra contra 10.327.255 L em 72 dias. A média diária na safra atual foi de 209.733,13 L de etanol contra $143.434,097 \mathrm{~L}$ da safra passada, representado um incremento diário médio de aproximadamente $46 \%$.

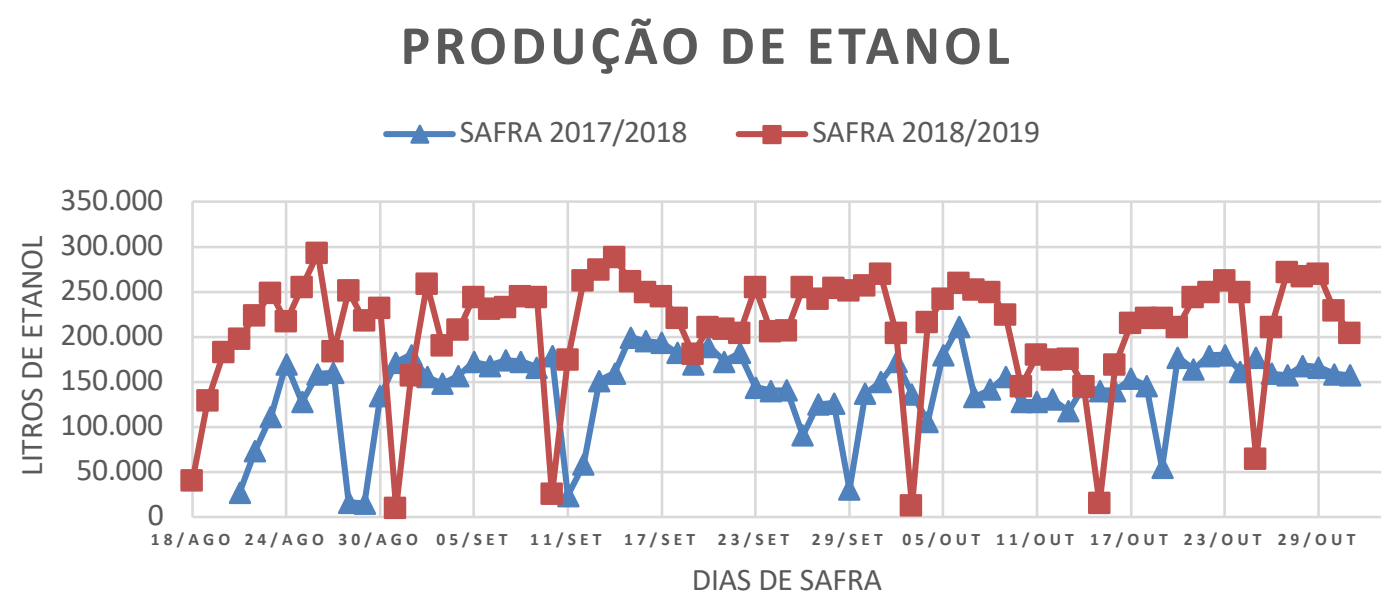

Figura 15: Comparativo da produção de etanol

Finalmente, todo este estudo mostrou que, com as modificações propostas, o sistema de fermentação tornou-se apto a receber mais matéria prima, mantendo a temperatura ótima de trabalho para a levedura, aumentou, consideravelmente, a capacidade de produção de etanol, o que representa um grande benefício econômico para a usina onde foi implementado. 


\section{CONCLUSÃO}

Com a previsão de uma safra mais voltada a produção de etanol, foi necessário estudar a planta de produção de etanol e identificar os pontos subdimensionados e propor a resolução do problema, com o mínimo de investimento financeiro.

Para o aumento da produção de etanol, verificou-se a necessidade de aumentar a quantidade de matéria-prima destinada a este fim. Entretanto, tal ação geraria um problema de superaquecimento da fermentação, o que motivou o redimensionamento do sistema de refrigeração das dornas.

Foi observado que, a área de transferência de calor nos estágios iniciais eram insuficientes para atender a condição desejada, portanto, as placas dos trocadores de calor foram redistribuídas, a fim de atender a necessidade do setor.

A ampliação do segundo estágio por conjugação de duas dornas, além de aumentar a capacidade de absorção de calor, proporcionou um tempo de retenção maior, favorecendo a conversão dos açúcares em etanol neste estágio.

Outro ponto relevante é a redução do custo de manutenção e operação do sistema, devido à retirada de operação de duas bombas e dois motores e o estabelecimento de uma reserva técnica de placas do modelo R56.

Por fim, o objetivo principal foi atingido. A produção de etanol no mesmo período foi $52 \%$ maior que na safra anterior, tendo como um dos principais responsáveis para este incremento as modificações propostas neste trabalho.

\section{REFERÊNCIAS BIBLIOGRÁFICAS}

Caldas, C. (1998). Manual de Análises Selecionadas Para Indústrias Sucroalcooleiras. 1.ed. Maceió: STAB, Capítulo 11 - Vinho Levurado e delevurado, leite tratado e fundo de dorna, p.149-156.

Companhia Nacional De Abastecimento (CONAB). (2018). Acompanhamento da Safra Brasileira de Cana-de-açúcar - Safra 2018/2019 - Segundo levantamento. Disponível em: <https://www.conab.gov.br/info-agro/safras/cana>. Acesso em: 7 de novembro de 2018.

Cunha, A., Missawa, L. G.; Reis, S. F.; Pereira, G. (2006). Control by sugar of Saccharomyces cerevisiae focculation for industrial ethanol production. FEMS YEAST RESEARCH, v. 6, p. 208-287.

Dryden Aqua. Scotland. Plate Heat Exchanger Operating \& Instruction Manual. Disponível em: <http://www.drydenaqua.com/heating_cooling/>. Acesso em: 25 de outubro de 2018.

Gut, J. A. W. (2003). Configurações ótimas para trocadores de calor a placas. 2003. p.244. Tese (Doutorado em Engenharia Química) - Departamento de Engenharia Química. Escola Politécnica da Universidade de São Paulo. São Paulo.

Hewitt, G. F.; Shires, G. L.; Bott, T. R. (1994). Process Heat Transfer. Boca Raton: CRC Press, Chapter 8 - Plate and Frame Heat Exchangers, p. 207-241.

Incropera, F. P.; Dewitt, D. P. 9 (2008). Fundamentos de transferência de calor e de massa. 6.ed. Rio de Janeiro: LTC, Capítulo 11 - trocadores de calor p. 424-442. 
Kakaç, S.; Liu, H.; Pramuanjaroenkij, A. (2012) Heat Exchangers: Selection, Rating and Thermal Design. 3.ed. Boca Raton: CRC Press. Chapter 2 - Basic Design Methods of Heat Exchangers, p. 48-101.

Leão, R. M.; Amorim, H. V. (2005). Fermentação Alcoólica: ciência e tecnologia. 1.ed. Piracicaba: Fermentec, Capítulo 3 - Avanços da Ciência e a Fermentação Alcoólica, p. 74-97.

Manoel, A. A. S.; Moraes, M. B. Da C.; Santos, D. F. L.; Neves, M. F. (2017). Determinants of corporate cash holdings in times of crisis: insights from Brazilian sugarcane industry private firms. International Food and Agrobusiness Management Review, v.21. n.2. p. 1-18.

Rein, P. (2008). Engenharia do açúcar de cana. 1.ed. Berlim: Bartens, Capítulo 21-Movimentação e estocagem do melaço, p. 571-582.

Souza, P. J.; Prado C. D.; Eleutherio, E. C.; Bonatto, D.; Malavazi, I.; Cunha, A. F. (2018). Improvement of Brazilian bioethanol production - challenges and perspectives on the identification and genetic modification of new strains of Saccharomyces cerevisiae yeasts isolated during ethanol process. Fungal Biology, v. 122, n. 6, p. 583-591.

Techaparin, A.; Thanonkeo, P.; Klanrit, P. (2017). High-temperature ethanol production using thermotolerant yeast newly isolated from Greater Mekong Subregion. Brazilian journal of Microbiology, v. 48, n. 3, p. 461-475.

Wang, P.; Zheng, D.; Chi, X.; Li, O.; Qian, C.; Liu, T.; Zhang, X.; Du, F.; Sun, P.; Qu,A.; Wu, X. (2014). Relationship of trehalose accumulation with ethanol fermentation in industrial Saccharomyces cerevisiae yeast strains. Bioresource Technology, v. 152, p. 371-376.

Zabed, H.; Faruq, G.; Sahu, J. N.; Azirun, M. S.; Hashim, R..; Boyce, A. N. (2014). Bioethanol Production from Fermentable Sugar Juice. The Scientific World Journal. http://dx.doi.org/10.1155/2014/957102. p. 1-11. 